\title{
A Study on the Intervention Model of Gastrointestinal Reactions Based on Self-efficacy Theory in Lung Cancer Patients During Chemotherapy
}

\author{
Liu Guixia ${ }^{1, ~ *, ~ W u ~ C a i y u n ~}{ }^{2}$, Sun Shenghong ${ }^{3}$, Zhang Hui ${ }^{1}$, Meng Yun ${ }^{1}$ \\ ${ }^{1}$ Department of Respiratory and Critical Care Medicine, First Affiliated Hospital of Anhui Medical University, Hefei, China \\ ${ }^{2}$ Department of Hemodialysis, First Affiliated Hospital of Anhui Medical University, Hefei, China \\ ${ }^{3}$ Organ Transplantation Center, First Affiliated Hospital of Anhui Medical University, Hefei, China
}

\section{Email address:}

386901459@qq.com (Liu Guixia),635376194@qq.com (Wu Caiyun), 179035620@qq.com (Sun Shenghong),

2761145197qq.com (Zhang Hui), 2740032073qq.com (Meng Yun)

${ }^{*}$ Corresponding author

\section{To cite this article:}

Liu Guixia, Wu Caiyun, Sun Shenghong, Zhang Hui, Meng Yun. A Study on the Intervention Model of Gastrointestinal Reactions Based on Self-efficacy Theory in Lung Cancer Patients During Chemotherapy. International Journal of Clinical Oncology and Cancer Research.

Vol. 6, No. 1, 2021, pp. 1-9. doi: 10.11648/j.ijcocr.20210601.11

Received: December 5, 2020; Accepted: December 19, 2020; Published: January 12, 2021

\begin{abstract}
Background: Although there are many studies on clinical intervention of gastrointestinal reactions induced by chemotherapy, the methods and means of intervention are inconsistent and the results differ greatly. More importantly, there are fewer relatively uniform intervention models with theoretical support. Therefore, based on the self-efficacy theory and its framework, the aim of this study was to construct an intervention model of gastrointestinal reactions in patients with lung cancer undergoing chemotherapy and evaluate its effects of clinical application. Methods: Based on self-efficacy theory, a intervention model of gastrointestinal reactions was constructed and applied to 30 patients with lung cancer who had gastrointestinal reactions during chemotherapy for three consecutive chemotherapy cycles. Results: After three cycles of intervention, the analysis of repeated measurement data showed that there was decreased in the total score of gastrointestinal reactions induced by chemotherapy, as well as in yield and psychological distress, and at the same time, increased in the scores of self-efficacy, objective support, support utilization and internal control $(\mathrm{P}<0.05)$. Conclusion: The intervention model based on the self-efficacy theory is feasible to intervene in the gastrointestinal reactions of patients with lung cancer during chemotherapy. It can improve their self-efficacy and effectively reduce the gastrointestinal reactions.
\end{abstract}

Keywords: Self-efficacy, Chemotherapy, Lung Cancer, Gastrointestinal Reactions

\section{Introduction}

Lung cancer is the most common cancer and the leading cause of cancer-related death in the world $[1,2]$. More than $80 \%$ of patients are stage III to IV at diagnosis [3]. Chemotherapy is an indispensable means in their comprehensive treatment, while gastrointestinal reactions are the most frequent and painful side effects [4-6]. Severe gastrointestinal reactions may reduce the quality of life of patients, affect the effectiveness of treatment, and even life-threatening. These reactions are not only related to chemotherapy drugs, but also closely related to the physiological, psychological and social status of patients. In today's shortage of nursing resources, it is a problem worthy of consideration for each medical and health personnel that how to mobilize patients to actively participate in their own disease management in order to reduce the symptoms and a series of psychological and social problems caused by the disease and its treatment.

As a kind of personal resources, self-efficacy can promote patients to improve the management ability of cancer and other chronic diseases, and can be adjusted according to the specific situations [7]. It is the core of the physical and psychological ability of cancer patients to manage their health [8]. There are many clinical intervention studies on gastrointestinal reactions caused by chemotherapy, but the 
intervention methods and means are inconsistent, and the results are quite different. What's more, there are fewer relatively uniform intervention models with theoretical support. Systematically studying the basis of human behavior under the theoretical guidance can significantly improve our understanding of cancer-related health behaviors and help design programs to improve these ones [9]. Therefore, based and framed on the self-efficacy theory, the aims of this study were to construct an intervention model of gastrointestinal reactions and evaluate its effects of clinical application in lung cancer patients undergoing chemotherapy.

\section{Model Construction}

\subsection{Theoretical Basis of the Model Construction}

The theory of self-efficacy derives from the viewpoint of triadic reciprocal determinism [10]. It is the foundation of social cognitive theory, which refers to the interaction and mutual determination of human behavior, intrinsic factors and environment, and is in the state of interactive causation [11]. In particular, Bandura emphasized that people's intrinsic factors and their behavioral outcomes played a decisive role in behaviors and environments. People can adjust their behaviors according to the constantly changing environment to obtain the desired results and avoid adverse outcomes or aversive emotional experience, while self-efficacy plays a certain role in regulating expected behaviors and environments [12].

Self-efficacy refers to the expectation or confidence of an individual to effectively cope with and successfully accomplish a certain task [13], which is related to the target of reference and the ability to judge in a specific situation, emphasizes the subjective initiative of human beings and the belief of their own abilities, and focuses on the influence of self-efficacy on the situation-specific behavior and decision-making [11]. It exerts the principal actions through the individual's cognitive, motivational, affective, and selective processes [14], and is essentially a subjective perception of self-ability, of which the most central and practical significance is the sense of self-efficacy.

The sense of self-efficacy is "the degree to which people feel confident that they can perform a task with the skills they have" [15], which is divided into the result expectation of individual independent speculation and the efficacy expectation of one subjective judgment, and is mainly established through the efficacy information provided by 4 information sources namely direct experience, vicarious experience, verbal persuasion, physiological and emotional state [10]. The higher a person's self-efficacy expectations are, the more active their behavior would be, as well as the more persistent their efforts and the more positive their emotional responses.

Since the self-efficacy theory was put forward, it has gradually crossed over from the initial education and mental health to chronic disease care, cancer screening and symptom management, etc., and the intervention practice is mainly carried out on various self-management projects based on this theory. As a positive psychological resource [16], self-efficacy has been paid more and more attentions in the application research of life-threatening diseases, including cancer. Previous study has shown that self-efficacy can enhance self-management behavior [17]. Cancer patients with higher self-efficacy are more likely to adopt healthier behaviors (such as regular exercise, active communication with health care providers), have a longer-lasting desire to maintain mental health (easy adaptation, less psychological distress), and meanwhile have fewer physical symptoms (less pain, less fatigue) and higher quality of life [18].

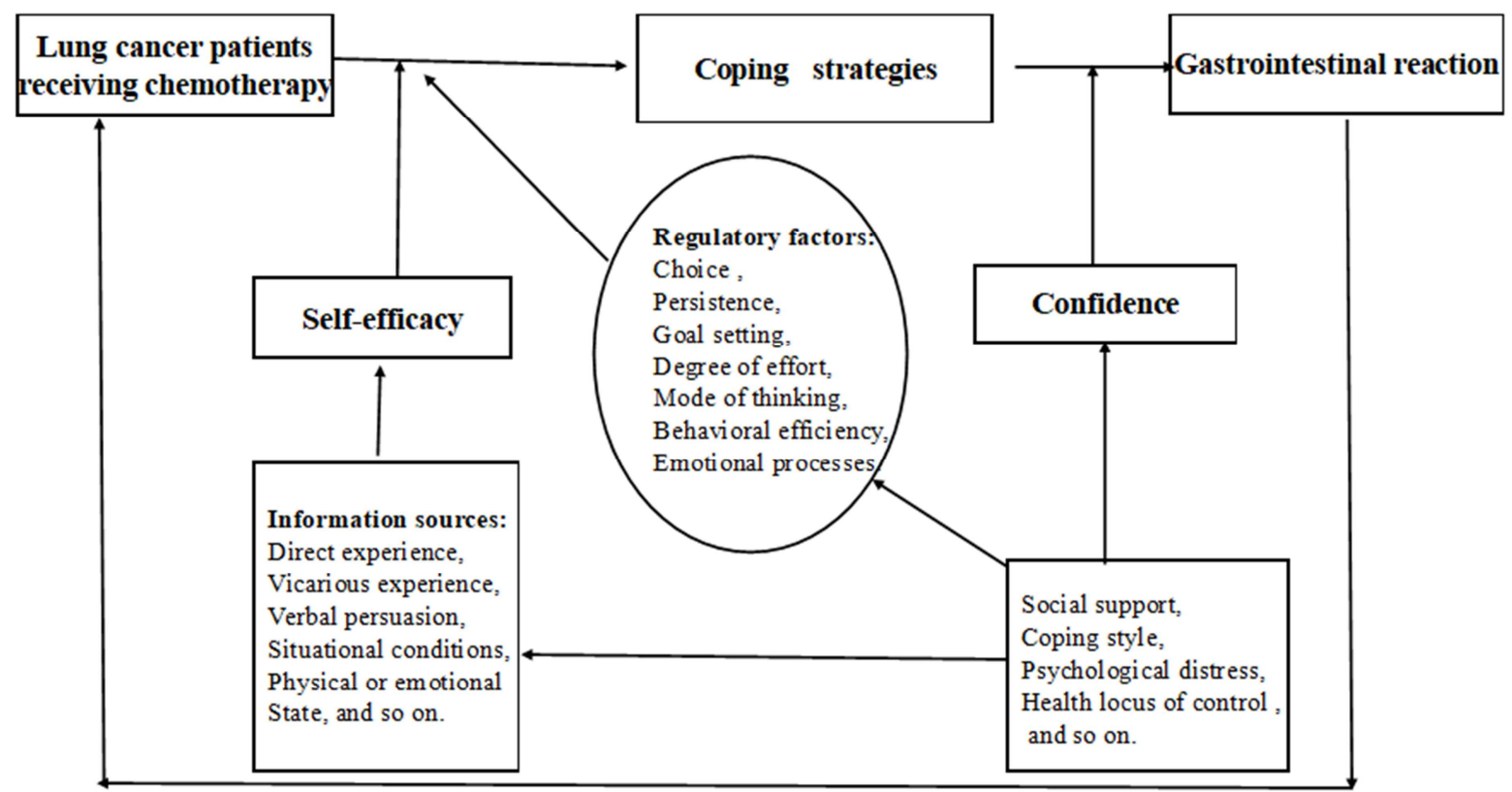

Figure 1. Preliminary theoretical model of self-efficacy-based intervention in gastrointestinal reactions of lung cancer patients undergoing chemotherapy. 


\subsection{Clinical Basis of the Model Construction}

Relevant literature and previous studies of this research group [19-21] showed that the incidences of anxiety and depression were high in lung cancer patients who had poor psychosomatic state, obvious psychological distress and low level of self-efficacy during chemotherapy, and at the same time, there were many problems such as insufficient social support, negative coping style, reduced internal control, and the occurrence of gastrointestinal reactions was related to them. Therefore, this study takes these as the starting points of intervention to develop the following theoretical prototype of the intervention model.

\subsection{Process and Content of the Model Construction}

Through semi-structured qualitative interview, the real feelings of the patients were deeply explored and the causes of the above problems were analyzed. After brainstorming discussion by 10 nursing staff $(9$ in respiratory department and 1 in oncology department, all of them had more than 15 years of work experience; among them, 5 were deputy chief nurses and 5 were in charge of nurses), the final specific intervention program and intervention model were as follows:

\subsubsection{Specific Intervention Programs}

i. Intervention Team

The intervention team was composed of responsible physicians, responsible nurses, psychological counselors, dietitians and researchers who participated in multiple disciplines and were uniformly trained. Network information personnel provided network information guarantee.

ii. Intervention Objectives

The intervention objectives included increasing social support and internal control of lung cancer patients undergoing chemotherapy, changing their adverse coping style, and reducing the level of psychological distress, so as to enhance their self-efficacy level to reduce the gastrointestinal reactions caused by chemotherapy.

iii. Intervention Object

The intervention object was mainly lung cancer patients with gastrointestinal reactions induced by chemotherapy.

iv. Intervention Time

It was during hospitalization and 2-3 weeks after discharge. v. Intervention Pathways

There were various forms of intervention, including propaganda and education handbook, teaching by PPT, personalized health education, micro-class, Wechat, cloud follow-up and telephone call-back, etc.

vi. Intervention Cycle

(1) During the first chemotherapy: The intervention theme was mainly direct experience acquisition of self-efficacy. Responsible physicians and nurses interviewed patients daily during hospitalization, gave them personalized health education and randomly intervened as appropriate to teach them to master 2-3 behavioral therapies, and gave a centralized lecture in the form of PPT to teach the knowledge and behavior therapy related to lung cancer chemotherapy.

(2) During the second chemotherapy: The main theme of the intervention was mainly to persuade, advise and increase patients' vicarious experience, including increasing patients' family and social support, changing them unhealthy coping styles, encouraging to interact in a variety of ways, organizing patients' communication meeting which the purpose was to setting an example role to convince them that they have the ability and confidence to cope with cancer and its treatment-related side effects.

(3) During the third chemotherapy: The main theme of the intervention is emotional counseling, including a intensive PPT lecture to explain the methods of emotional relief (such as walking, listening to music, chatting, watching TV, keeping emotional diaries and cards, etc.), a patients' communication meeting organized to make them communicate their own psychological course after cancer, how to overcome negative emotions and their methods, and setting an example again.

vii. Follow-up after Discharge

(1) Being familiar with the patient's cancer pathological stage, chemotherapy regimen and main problems during chemotherapy before the follow-up.

(2) Assessing the changes in diet, sleep, urination, defecation and appetite or other uncomfortable problems of patients after discharge from the hospital.

(3) Evaluating the side effects of chemotherapy such as nausea, vomiting, abdominal distension, diarrhea, constipation, etc. listening to patients' chief complaints and extract important information.

(4) Assessing the psychological and emotional status of patients and know whom they live together with. What was the most worrying issue at present? What were the psychological stresses and needs? What support was the most needed? Listening to patients' complaints and extract important information, and giving the appropriate intervention according to the specific situations.

(5) Comprehensive evaluating of gastrointestinal reactions and continuous health educating.

viii. Effect Evaluation Index

The effectiveness evaluation indicators included psychological measurement index (social support, coping style, types of locus of control, psychological distress and self-efficacy) and gastrointestinal reactions. 


\subsubsection{Intervention Model}

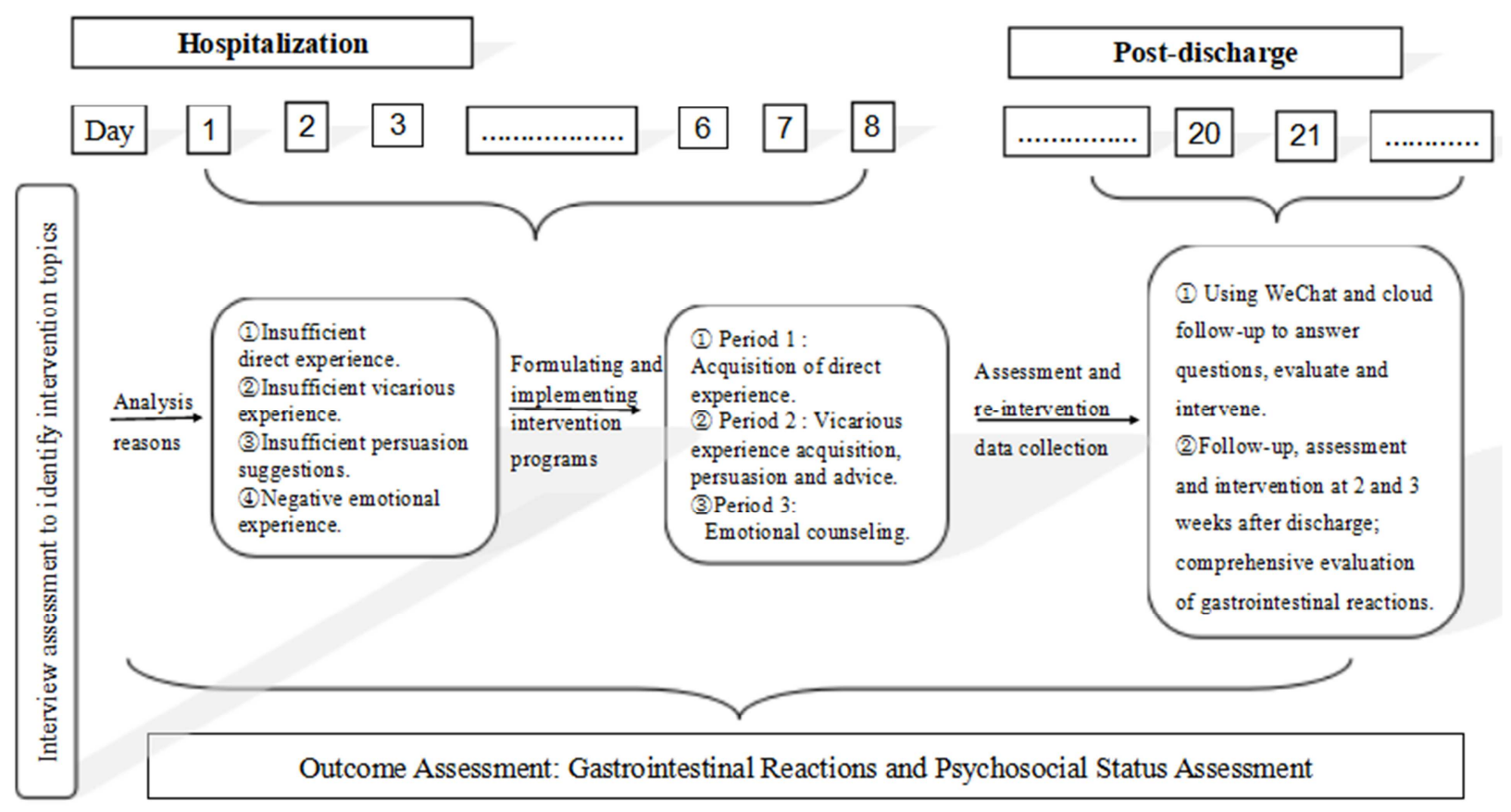

Figure 2. Intervention model of gastrointestinal reactions based on self-efficacy theory in lung cancer patients undergoing chemotherapy.

\section{Clinical Application}

\subsection{Materials and Methods}

\subsubsection{Study Subjects}

By convenience sampling method, lung cancer patients undergoing chemotherapy were selected and divided into two groups according to SPSS random number generator at the department of respiratory medicine of a grade III-A hospital in Anhui province from January 2019 to September 2020, and one group was set as intervention group, the other was as control group. Patients inclusion criteria: (1) Patients were primary bronchogenic carcinoma undergoing first chemotherapy. (2) Being lucid, age $\geq 18$ years old. (3) Gastrointestinal reactions score of initial evaluation $(\geq 1)$, and patients expected to survive for more than 3 months and can persist in completing at least 3 consecutive rounds of chemotherapy. (4) Patients were aware of the condition and willing to participate in this study with good compliance. Patients exclusion criteria: (1) Patients were intracranial hypertension caused by intracranial metastasis of lung cancer. (2) Patients had a history of gastrointestinal illness and mental illness, as well as verbal communication disorders. 3 patients in the control group were dropped out of the study ( 2 lost visits, 1 suspended chemotherapy for some reasons), and 2 patients in the intervention group were withdrew from the study (1 lost to follow-up, 1 changed to off-site treatment). The final effective sample size of the intervention group was 30 , with an effective rate of $93.75 \%$, while 29 patients in the control group had an effective rate of $90.63 \%$.

There were no significant differences in the basic conditions of the two groups and scores of gastrointestinal reactions, social support, coping style, health locus of control, psychological distress and general efficacy $(P>0.05)$, which were comparable, as shown in Table 1 and Table 2.

Table 1. Comparison of basic conditions between the two groups.

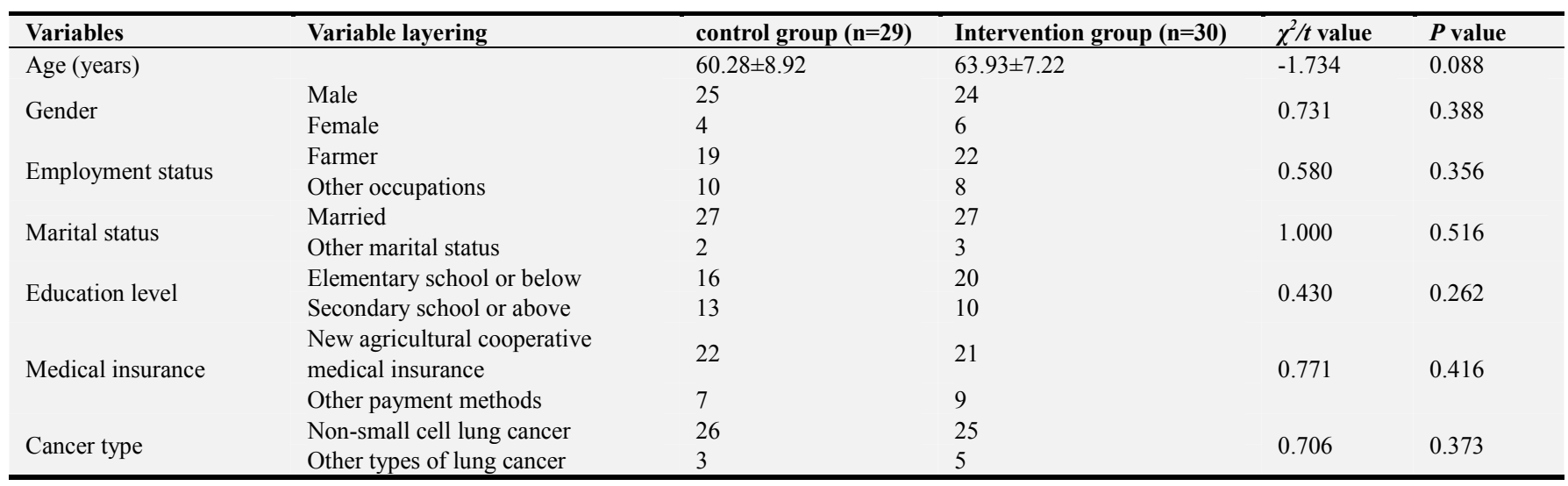


Table 2. Comparison of gastrointestinal reactions, social support, coping style, health locus of control, psychological distress and general efficacy between the two groups.

\begin{tabular}{|c|c|c|c|c|}
\hline Variables & Intervention group $(n=30)$ & Control group $(n=29)$ & $T$ value & $P$ value \\
\hline \multicolumn{4}{|l|}{ Social support } & 0.998 \\
\hline Subjective support & $19.33 \pm 4.06$ & $19.55 \pm 3.93$ & 0.339 & 0.736 \\
\hline Objective support & $8.30 \pm 2.55$ & $7.83 \pm 2.04$ & -0.785 & 0.436 \\
\hline Support utilization & $6.30 \pm 2.55$ & $6.03 \pm 1.27$ & -0.509 & 0.613 \\
\hline Total support score & $33.80 \pm 7.27$ & $33.41 \pm 5.88$ & -0.224 & 0.824 \\
\hline \multicolumn{5}{|l|}{ Coping style } \\
\hline Face & $15.00 \pm 2.79$ & $16.14 \pm 2.77$ & 1.630 & 0.109 \\
\hline Yield & $10.33 \pm 3.17$ & $9.38 \pm 2.54$ & -1.274 & 0.208 \\
\hline Avoid & $15.03 \pm 2.30$ & $15.17 \pm 1.16$ & 0.194 & 0.847 \\
\hline \multicolumn{5}{|l|}{ Health Locus of Control } \\
\hline Chance & $17.83 \pm 3.11$ & $19.45 \pm 4.00$ & 1.743 & 0.087 \\
\hline Internal control & $16.53 \pm 4.15$ & $17.93 \pm 3.08$ & 1.465 & 0.147 \\
\hline Impact of others & $27.27 \pm 4.01$ & $25.90 \pm 4.39$ & -1.252 & 0.216 \\
\hline Psychological distress & $4.90 \pm 1.86$ & $4.21 \pm 1.45$ & -1.591 & 0.117 \\
\hline General efficacy & $2.03 \pm 0.20$ & $2.07 \pm 0.19$ & 1.024 & 0.310 \\
\hline
\end{tabular}

\subsubsection{Research Methods}

Patients in the control group were given routine nursing measures according to the health education path table formulated by the department (including: guidance on going in and out of the hospital, nursing for resting position, diet, medication, laboratory examination, pipeline and safety, and education of disease-related knowledge during hospitalization, etc.), while patients in the intervention group were given interventions according to the above intervention model on the basis of routine nursing.

\subsubsection{Outcome Evaluations}

i. Gastrointestinal Reactions

Gastrointestinal reactions of patients were evaluated by the chemotherapy gastrointestinal symptom scale developed by He Haiyan in 2011. The scale includes 10 gastrointestinal symptom occurrence items and 4 symptom influence items, which constitute two dimensions of symptom occurrence and symptom influence. Higher scores imply more severe symptoms and greater impact on life [22]. The Cronbach's $\alpha$ coefficient of the total scale and its two dimensions were 0 . $81,0.82$ and 0.91 in this study.

ii. Social Support

Patients' social support was assessed with Social Support Rating Scale, which has 10 items, including subjective support, objective support and social support utilization, and higher scores indicate more support [23]. The Cronbach's $\alpha$ coefficient of the scale ranged from 0.85 to 0.89 in this study.

iii. Coping Style

Medical Coping Modes Questionnaire was used to assess coping styles of patients. It consisted of 20 items, including three subscales of facing, avoiding and yielding, and higher scores represent that the individual is more likely to adopt this coping style [24]. The Cronbach's $\alpha$ coefficient of each subscale was $0.61,0.65$ and 0.63 in this study.

iv. Types of Locus of Control

The types of locus of control were assessed with Multidimensional Health Locus of Control Scale compiled by Wallston in 1994 . The scale has 18 items, including three dimensions of internal control, impact of others and chance, which the latter two are known as external locus of control, and higher score of each dimension indicates the individual is more inclined to be the type of locus of control [25]. The Cronbach's $\alpha$ coefficient of the three subscales were $0.51,0$. 55 and 0.63 in this study.

\section{v. Psychological Distress}

Patients' distress was measured with Distress Thermometer that a visual analogue scoring tool shaped like a thermometer, which is used a scale of $0-10$ to represent the degree of psychological distress. Higher score means individual being more painful, and a score greater than 4 represents a clinically significant level of discomfort [26].

vi. Self-efficacy

The self-efficacy level of patients was assessed by the Chinese version of General Self-Efficiency Scale. The table consists of 10 items to form a dimension with good reliability and validity [27]. A higher score indicates a stronger sense of patient's self-efficacy. In this study, the Cronbach's $\alpha$ coefficient of the scale was 0.81 .

\subsubsection{Statistical Analysis Methods}

SPSS 17.0 software was used for statistical analysis. The data of the two groups before the intervention were compared by $\mathrm{t}$-test $/ \mathrm{x}^{2}$-test, while the ones after intervention were analyzed by variance analysis of repeated measurement data. The test level was $\alpha=0.05$, and $P \leq 0.05$ was statistically significant.

\subsection{Results}

After three consecutive chemotherapy cycles, variance analysis of repeated measurement data showed that the observation indexes (except for the chance dimension of health locus of control) of the two groups were affected by time, and some of them had interactions between time and group. The changes were different between the two groups in general efficacy, coping style, type of health locus of control, social support, psychological distress and gastrointestinal reactions, which had statistical significance after intervention $(\mathrm{P}<0.05)$, as shown in Table 3, Table 4, Table 5 and Table 6. 
Liu Guixia et al:: A Study on the Intervention Model of Gastrointestinal Reactions Based on Self-efficacy

Theory in Lung Cancer Patients During Chemotherapy

Table 3. Comparison of variance analysis of social support repeated measurement data between two groups after interventions.

\begin{tabular}{llllll}
\hline Time & Group & Subjective support & Objective support & Support utilization & Total support score \\
\hline \multirow{2}{*}{ First time } & Control group & $19.31 \pm 3.91$ & $7.80 \pm 2.04$ & $6.13 \pm 1.27$ & $33.47 \pm 5.28$ \\
& Intervention group & $19.20 \pm 4.04$ & $8.30 \pm 2.55$ & $6.32 \pm 2.15$ & $33.98 \pm 7.21$ \\
Second time & Control group & $22.79 \pm 4.80$ & $8.21 \pm 2.34$ & $6.45 \pm 1.90$ & $37.45 \pm 6.83$ \\
& Intervention group & $22.57 \pm 5.29$ & $9.83 \pm 2.10$ & $7.13 \pm 2.39$ & $39.87 \pm 7.23$ \\
Third time & Control group & $24.72 \pm 5.42$ & $9.67 \pm 2.13$ & $7.10 \pm 1.80$ & $41.48 \pm 6.34$ \\
& Intervention group & $26.00 \pm 4.03$ & $10.37 \pm 1.71$ & $8.60 \pm 1.78$ & $44.33 \pm 5.63$ \\
F value (time) / P value & $44.300 / 0.000$ & $18.756 / 0.000$ & $19.581 / 0.000$ & $60.693 / 0.00$ \\
F value (Group) / P value & $0.083 / 0.774$ & $4.763 / 0.033$ & $4.084 / 0.048$ & $1.965 / 0.166$ \\
F value (Interactive) / P value & $0.943 / 0.336$ & $2.542 / 0.088$ & $3.300 / 0.041$ & $0.971 / 0.385$ \\
\hline
\end{tabular}

Table 4. Comparison of variance analysis of coping style repeated measurement data between two groups after interventions.

\begin{tabular}{lllll}
\hline Time & Group & Face & Avoid & Yield \\
\hline \multirow{2}{*}{ First time } & Control group & $16.16 \pm 2.27$ & $15.37 \pm 1.13$ & $9.48 \pm 2.14$ \\
& Intervention group & $15.90 \pm 2.79$ & $15.33 \pm 2.31$ & $10.13 \pm 3.07$ \\
Second time & Control group & $16.14 \pm 4.27$ & $16.24 \pm 2.21$ & $9.72 \pm 3.24$ \\
& Intervention group & $18.23 \pm 3.84$ & $15.64 \pm 2.30$ & $8.03 \pm 2.34$ \\
Third time & Control group & $16.87 \pm 4.28$ & $16.55 \pm 2.25$ & $8.79 \pm 2.54$ \\
& Intervention group & $19.17 \pm 4.50$ & $16.20 \pm 2.51$ & $6.07 \pm 1.31$ \\
F value (time) / P value & & $9.325 / 0.000$ & $7.741 / 0.001$ & $23.664 / 0.000$ \\
F value (Group) / P value & $1.349 / 0.250$ & $1.156 / 0.287$ & $4.722 / 0.034$ \\
F value (Interactive) / P value & $8.428 / 0.001$ & $2.111 / 0.131$ & $14.101 / 0.000$ \\
\hline
\end{tabular}

Table 5. Comparison of variance analysis of repeated measurement data of health locus of control between two groups after interventions.

\begin{tabular}{lllll}
\hline Time & Group & Chance & Internal control & Impact of others \\
\hline \multirow{2}{*}{ First time } & Control group & $19.46 \pm 4.01$ & $17.19 \pm 3.08$ & $25.90 \pm 4.39$ \\
& Intervention group & $18.83 \pm 3.11$ & $16.53 \pm 4.15$ & $26.97 \pm 4.11$ \\
Second time & Control group & $17.72 \pm 3.40$ & $15.76 \pm 2.95$ & $25.72 \pm 3.61$ \\
& Intervention group & $20.40 \pm 4.09$ & $17.23 \pm 3.07$ & $24.33 \pm 3.40$ \\
Third time & Control group & $18.93 \pm 3.92$ & $17.72 \pm 3.86$ & $24.86 \pm 2.78$ \\
& Intervention group & $19.13 \pm 4.88$ & $21.23 \pm 4.86$ & $24.27 \pm 6.14$ \\
F value (time) / P value & $0.225 / 0.791$ & $16.074 / 0.000$ & $4.858 / 0.011$ \\
F value (Group) / P value & $0.445 / 0.507$ & $4.274 / 0.043$ & $0.075 / 0.785$ \\
F value (Interactive) / P value & $4.732 / 0.011$ & $4.597 / 0.014$ & $2.198 / 0.118$ \\
\hline
\end{tabular}

Table 6. Comparison of variance analysis of repeated measurement data of psychological distress, general efficacy and gastrointestinal reactions between two groups after interventions.

\begin{tabular}{lllll}
\hline Time & Group & psychological distress & general efficacy & Total score of gastrointestinal reactions \\
\hline \multirow{2}{*}{ First time } & Control group & $4.41 \pm 1.44$ & $2.09 \pm 0.19$ & $5.97 \pm 1.11$ \\
& Intervention group & $4.70 \pm 1.16$ & $2.03 \pm 0.20$ & $5.97 \pm 2.34$ \\
Second time & Control group & $4.07 \pm 1.22$ & $2.22 \pm 0.15$ & $4.72 \pm 2.31$ \\
& Intervention group & $3.47 \pm 0.86$ & $2.32 \pm 0.19$ & $2.83 \pm 1.66$ \\
Third time & Control group & $3.93 \pm 0.96$ & $2.25 \pm 0.25$ & $3.72 \pm 1.67$ \\
& Intervention group & $3.03 \pm 1.26$ & $2.78 \pm 0.30$ & $1.63 \pm 0.67$ \\
F value (time) / P value & $25.703 / 0.000$ & $83.869 / 0.000$ & $76.214 / 0.000$ \\
F value (Group) / P value & $4.407 / 0.040$ & $17.756 / 0.000$ & $13.712 / 0.000$ \\
F value (Interactive) / P value & $17.129 / 0.000$ & $35.761 / 0.000$ & $8.662 / 0.000$ \\
\hline
\end{tabular}

\subsection{Discussion}

\subsubsection{Intervention Model of Gastrointestinal Reactions Based on Self-efficacy Theory Can Improve Psychological Status of Lung Cancer Patients}

When lung cancer is diagnosed, most patients are in the middle or advanced stage, and chemotherapy is the main treatment method. Despite the continuous optimization of chemotherapy regimens and the continuous development of new anticancer drugs, the toxic and side effects related to chemotherapy and its auxiliary drugs are still relatively large, and the long-term therapeutic effect of lung cancer is still unsatisfactory and the prognosis is poor. In addition, there is often the thought of attaching importance to treatment and neglecting nursing care in clinical practice. Therefore, patients often have a weak sense of self-management of disease, poor ability of self-care, easy to feel pessimistic, disappointed, and poor quality of life. Due to the low acceptance and utilization of care and help provided by family and society, they are prone to adopt negative coping styles such as yielding, greater negative emotions, obvious psychological distress and lower internal control, attributing their health problems more to influential others or some controlled factors, relying more on others in the treatment process of the disease, and having weak 
belief and poor ability to fight cancer. So, while constantly optimizing the chemotherapy regimens for lung cancer and paying attention to treatment, it is particularly important for nursing to explore how to enhance patients' confidence in anti-cancer, awareness of cancer management and improve the ability of self-management of cancer.

The sense of self-efficacy is the degree of confidence that an individual can use his/her own skills to complete a specific task. It affects the thinking mode and emotional response mode of an individual in the process of task execution, and determines the selectivity of an individual for behavioral task as well as the degree of effort and persistence of the task. A research had shown that patients with high self-efficacy had a more positive attitude toward life, which was of great significance for promoting mental health [28]. This study also showed that through the intervention based on the above model, there were decreasing in the scores of yield and psychological distress as well as the increasing in the scores of objective support, support utilization and internal control $(P<0.05)$, indicating that the model based on self-efficacy theory to intervene in gastrointestinal reactions of lung cancer patients with chemotherapy can effectively alleviate patients' negative emotions and maintain their mental health level.

At the same time, the researches also shown that the high level of social support reflects the good interpersonal relationship of the individual, which can change the individual's view on the fearful event, reduce the individual anxiety, and as a direct facilitator of self-efficacy, increasing the social support can improve individual's self-efficacy and the ability to deal with the disease [28-30]. Positive coping style means that individuals may have better ability and skills to manage diseases, be more active and willing to participate in treatment, and thus have higher compliance with treatment and nursing. While yielding is a negative coping strategy, which will aggravate patients' psychological distress and condition, and is not conducive to the treatment and rehabilitation of diseases [31, 32]. Individuals with an internal locus of control believe they have the ability to control their health problems and are more likely to seek help from a professional psychologist, whereas those with an external locus of control are less likely to do so $[33,34]$. Based on the health locus of control, psychological distress, coping style and social support of the patients, combined with semi-structured qualitative interview, this study explored some subjective and objective factors that influence the level of self-efficacy of the patients, then extracted specific and individualized intervention themes, developed individualized and targeted intervention measures and implemented them one by one. So patients can actively utilize social support network, correctly face cancer, improve the internal locus of control, increase confidence in coping with cancer and the anticipation of the ability to solve cancer-related problems, thus maintaining their mental health at a relatively ideal level to actively seek medical information, effectively manage adverse emotions, usefully reduce the level of psychological distress, obviously enhance medical compliance behavior and significantly improve their ability to self-manage cancer. This also indirectly reflects the feasibility and effectiveness of the clinical application of the intervention model of gastrointestinal reactions based on the self-efficacy theory in lung cancer patients during chemotherapy, which is worth further promotion and application.

\subsubsection{Intervention Model of Gastrointestinal Reactions Based on the Self-efficacy Theory Can Reduce Gastrointestinal Reactions of Lung Cancer Patients}

There are 4 sources of self-efficacy [35]: (1) Direct experience: successful execution of a task. (2) Vicarious experience: Seeing someone succeed in completing a task in a similar situation. (3) The individual can be persuaded or advised. (4) Adjustment of psychological response under special circumstances. Therefore, expanding any of the above sources of self-efficacy may enhance the level of individual's self-efficacy. The stronger the belief (expected efficacy) is, the more positive the patient's emotional response and behavior would be, and also the greater and more persistent the effort. A study had shown that the self-efficacy level of cancer patients was generally low [36], the intervention based on the self-efficacy theory can change patients' misconceptions and improve their ability to deal with the side effects of radiotherapy and chemotherapy [37]. This study was aimed at 4 sources of self-efficacy to intervene in order to enhance patients' confidence in anti-cancer and improve their ability to overcome or cope with side effects caused by chemotherapy. The results showed that the self-efficacy level of the patients was significantly improved $(P<0.05)$ in the intervention group, indicating that the intervention model of gastrointestinal reactions based on the self-efficacy theory could improve the sense of self-efficacy of lung cancer patients with gastrointestinal reactions induced by chemotherapy.

A positive self-belief and the sense of personal control are the resources for patients to cope effectively [8]. Compared with those with low self-efficacy, patients with high self-efficacy were more likely to implement some effective coping strategies in order to achieve good psychological expectations (psychological adaptation and improved quality of life) and medical outcomes (less disease-related symptoms and side effects) [8], and more confident that they have the ability to overcome difficulties [38], easier to perform certain health behaviors or cope with plights [39], and it can be adjusted according to the specific situation [7]. In this study, patients in the intervention group had an enhanced sense of self-efficacy after intervention, and those with a high level of self-efficacy had stronger anti-cancer beliefs and a strong desire to live. In the face of chemotherapy-induced adverse reactions such as loss of appetite, nausea, vomiting and constipation, instead of the past medical management model of being passively instilled, grudgingly accepted, and forced to collaboration, they believed that they have the ability to cope with and have positive expectations of outcomes, and at the same time, they actively took actions (becoming proactive in seeking and making effective use of social support systems and medical resources, increasing the frequency of effective communications between medical staff and patients, being easily persuaded or advised to perceive and deal with problems in the bud, actively learning the knowledge and a variety of behavioral skills of preventing 
gastrointestinal reactions caused by chemotherapy, properly managing bad emotions, purposefully avoiding unhealthy emotional distress, etc.), which made them show good behavior efficiency and strong self-management ability in the process of preventing and treating of gastrointestinal reactions caused by chemotherapy. Compared with the control group, except for the influence of the time factor and its interaction with the intervention, the total score of gastrointestinal reactions of the patients decreased with the improvement of self-efficacy in the intervention group $(P<0.05)$, which was consistent with the previous studies $[40,41]$. It shows that the intervention model based on self-efficacy theory can effectively reduce the gastrointestinal reactions of lung cancer patients undergoing chemotherapy, and at the same time, also reminds that the clinical medical staff should pay more attentions to the evaluation of self-efficacy and intervene in time for ones with poor self-efficacy.

\section{Conclusion}

To sum up, the intervention model of gastrointestinal reactions based on self-efficacy theory can effectively alleviate patients' negative emotions, reduce the level of psychological distress and improve the sense of self-efficacy by increasing their social support, changing the disease coping strategies and the type of locus of control, thus maintaining their psychology at a healthier level and reducing their gastrointestinal reactions while increasing self-efficacy. However, due to the limitation of time and for the facilitation of sampling, the selection of research subjects has a certain degree of bias. What's more, because of no long-term and in-depth dynamic study on such patients, future researches should be carried out in multiple centers and extend the study period to verify the above findings, so as to better serve the clinical practice.

\section{Conflict of Interest}

All the authors do not have any possible conflicts of interest.

\section{Acknowledgements}

With the support of the First Affiliated Hospital of Anhui Medical University, all data were collected in the Department of Respiratory and Critical Care Medicine of the hospital. Thanks to the director and head nurse for their support and the active cooperation of all the patients and their families. Special thanks to all nursing experts and colleagues who participated in this study.

\section{References}

[1] Sparla A, Flach-Vorgang S, Villalobos M, et al. Reflection of illness and strategies for handling advanced lung cancer - a qualitative analysis in patients and their relatives [J]. BMC Health Serv Res, 2017, 17 (1): 173. DOI: 10.1186/s12913-017-2110-x.
[2] $\mathrm{Gu} \mathrm{W}, \mathrm{Xu} Y M$, Zhong BL. Health-related quality of life in Chinese inpatients with lung cancer treatedin large general hospitals: across-sectional study [J]. BMJ Open, 2018, 8 (4): e019873. DOI: 10.1136/bmjopen-2017-019873.

[3] Wang D, Fu J. Symptom clusters and quality of life in China patients with lung cancer undergoing chemotherapy $[\mathrm{J}]$. Afr Health Sci, 2014, 14 (1): 49-55. DOI: 10.4314/ahs.v14i1.8.

[4] Koshiyama M, Matsumura N, Imai S, et al. Combination of Aprepitant, Azasetron, and Dexamethasone as Antiemetic Prophylaxis in Women with Gynecologic Cancers Receiving Paclitaxel/Carboplatin Therapy [J]. Med Sci Monit, 2017, 23: 826-833. DOI: $10.12659 / \mathrm{msm} .899741$.

[5] Wahlang JB, Laishram PD, Brahma DK, et al. Adverse drug reactions due to cancer chemotherapy in a tertiary care teaching hospital [J]. Ther Adv Drug Saf, 2017, 8 (2): 61-66. DOI: $10.1177 / 2042098616672572$.

[6] Xie J, Chen LH, Ning ZY, et al. Effect of transcutaneous electrical acupoint stimulation combined with palonosetron on chemotherapy-induced nausea and vomiting: a single-blind, randomized, controlled trial [J]. Chin J Cancer, 2017, 36 (1): 6. DOI: $10.1186 / \mathrm{s} 40880-016-0176-1$.

[7] Grimmett C, Haviland J, Winter J, et al. Colorectal cancer patient's self-efficacy for managing illness-related problems in the first 2 years after diagnosis, results from the ColoREctal Well-being (CREW) study [J]. J Cancer Surviv, 2017, 11 (5): 634-642. DOI: 10.1007/s11764-017-0636-x.

[8] Omran S, Mcmillan S. Symptom Severity, Anxiety, Depression, Self- Efficacy and Quality of Life in Patients with Cancer [J]. Asian Pac J Cancer Prev, 2018, 19 (2): 365-374. DOI: 10.22034/APJCP.2018.19.2.365.

[9] Atienza AA, Serrano KJ, Riley WT, et al. Advancing Cancer Prevention and Behavior Theory in the Era of Big Data $[\mathrm{J}]$. J Cancer Prev, 2016, 21 (3).

[10] Cai JK. On the self-efficacy of young nursing teachers [J]. Teacher, 2020, (5): 127-128.

[11] Ma J, Jia HZ (2019). Construction of Triadic Reciprocal Reading Promotion Model from the Perspective of Self-efficacy [J]. Library \& Information, 2019, (2): 46-56. DOI: 10.11968/tsyqb.1003-6938.2019024.

[12] Bandura A. The self system in reciprocal determinism [J]. American Psychologist, 1978, 33 (4): 344-358.

[13] Peterson AM, Harper FW, Albrecht TL, et al. Parent caregiver self-efficacy and child reactions to pediatric cancer treatment procedures [J]. J Pediatr Oncol Nurs, 2014, 31 (1): 18-27. DOI: $10.1177 / 1043454213514792$.

[14] Bandura A. Self-efficacy. The exercise of control [M]. New York: Freeman, 1997, pp. 116.

[15] Zhang Lei. A Study on the Intervention of Class Group Tutoring on Self-efficacy of Higher Vocational Students [J]. Journal of Shanxi Institute of Economic Management, 2018, 26 (002): 10-15.

[16] Baskin AS, Kwan L, Connor SE, et al. Low self-efficacy is associated with decreased emergency department use in underserved men with prostate cancer. Urologic Oncology Seminars \& Original Investigations, 2016, 34: 3. e15-21. 
[17] LI DJ, XING FM, DONG CY, et al. Long-term effects of nursing intervention based on self-efficacy theory on dietary self-management behavior of elderly diabetic patientss [J]. Chinese Nursing Research, 2020, 34 (5): 897-899. DOI: 10.12102/j.issn.1009-6493.2020.05.036.

[18] Huang FF, Yang Q, Wang AN, et al. Psychometric properties and performance of existing self-efficacy instruments in cancer populations: a systematic review [J]. Health Qual Life Outcomes, 2018, $16 \quad$ (1): 241. DOI: 10.1186/s12955-018-1066-9.

[19] Huang L, Li XL, Luo X, et al. Analysis of the levels of psychological distress and its influence factors in patients with lung cancer [J]. Chinese Journal of Practical Nursing, 2015, (35): 2704-2707. 10.3760/cma.j.issn.1672-7088.2015.35.018.

[20] Liu GX, Zhang XQ. Study on the relationship between social support and gastrointestinal reaction in patients with lungcancer during chemotherapy [J]. Chin J Prac Nurs, 2016, $32 \quad(30): \quad 2335-2339$. 2016.30 .004 DOI: 10.3760/cma.j.issn.1672-7088.2016.30.004.

[21] Liu GX, Zhang H, Meng Y, et al. A path analysis of impacts of psychological control source, psychological distress and psychosomatic status on the gastrointestinal symptoms of lung cancer patients undergoing chemotherapy [J]. Journal of Nurses Training, 2018, 33 (4): 305-309. DOI: 10.16821/j.cnki.hsjx.2018.04.005.

[22] He HY. The Construction of Assessment Tools and Clinical Research on Chemotherapy-Related Gastrointestinal Symptoms Burden [D]. Army Medical University, 2011.

[23] Wu J, Zhou J. The mediating effect of self-efficacy between social support and benefit finding among caregivers of hospitalized patients with cancer [J]. Nursing of Integrated Traditional Chinese and Western Medicine, 2020, 6 (1): 5-9. DOI: $10.11997 /$ nitcwm.202001002.

[24] Shen XH, Jiang QJ. Report on application of Chinese version MCMQ in 701 patients [J]. Chinese Journal of Behavioral Medicine and Brain Science, 2000, 9 (1): 18. DOI: 10.3760/cma.j.issn.1674-6554.2000.01.008.

[25] ZHU TT, LIU DL, ZHANG J, et al. Progress of Measurement Tools on Locus of Control [J]. Chinese General Practice, 2019, 22 (20): 2476-2481. 10.12114/j.issn.1007-9572.2018.00.384.

[26] Zhang YNg, Zhang HW, Song LL, et al. Application of the NCCN Distress Thermometer in Chinese cancer patients [J]. Chinese Mental Health Journal, 2010, 24 (12): 897-902.

[27] WANG CK, HU ZF, LIU Y. Evidences for Reliability and Validity of the Chinese Version of General Self-Efficacy Scale [J]. Chinese Journal of Applied Psychology, 2001, 7 (1): $37-40$.

[28] Zhao QQ, Yang ZH, Mi X, et al. Structural equation model of self-efficacy, social support, coping style and psychological resilience in lung cancer patients [J]. Chongqing Medical Journal, 2017, $46 \quad(30)$ : 4310-4312. DOI: 10.3969/j.issn.1671-8348.2017.30.048.

[29] Lee YS, Suchday S, Wylie-Rosett J. Perceived social support, coping styles, and Chinese immigrants' cardiovascular responses to stress [J]. Int J Behav Med2012, 19 (2): 174-185. DOI: $10.1007 / \mathrm{s} 12529-011-9156-7$.

[30] Huang FF, Yang Q, Zhang J, et al. The structural equation model on self-efficacy during post-op rehabilitation among non-small cell lung cancer patients $[\mathrm{J}]$. PLoS One, 2018, $13 \quad$ (9): $\quad$ e0204213. 10.1371/journal.pone.0204213.

[31] Liu XX, Liu SYi, Zhu Y, et al. Study on status quo of psychological control source, coping styles and psychological distress of lung cancer patients [J]. Chinese Nursing Research, 2015, (3): 324-326. 10.3969/j.issn.10096493.2015.03.024.

[32] Liu LL, Lin P, Zhao ZJ. A path analysis of impacts of self-efficacy, social support and coping style on resilience in acute myocardial infarction patients $[\mathrm{J}]$. Chinese Journal of Nursing, 2016, $51 \quad$ (1): 21-25. DOI: 10.3761/j.issn.0254-1769.2016.01.004.

[33] Hamilton JG, Lobel M. Psychosocial Factors Associated With Risk Perceptions for Chronic Diseases in Younger and Middle-Aged Women [J]. Women Health, 2015, 55 (8): 921-942. DOI: 10.1080/03630242.2015.1061094.

[34] Perenc L, Radochonski M. Psychological Predictors of Seeking Help from Mental Health Practitioners among a Large Sample of Polish Young Adults [J]. Int J Environ Res Public Health, 2016, 13 (11): 1-9. DOI: 10.3390/ijerph13111049.

[35] Lambert SD, Girgis A, Turner J, et al. A pilot randomized controlled trial of the feasibility of a self-directed coping skills intervention for couples facing prostate cancer: rationale and design [J]. Health Qual Life Outcomes, 2012, 10: 119. DOI: 10.1186/1477-7525-10-119.

[36] Qian D, Xu XJ, Fan LL, et al. Research Progress in the Relationship of Self-efficacy and the Quality of Cancer Patients' Lives in Foreign Countries [J]. Chinese General Practice, 2014, (36): 4286-4290. DOI: 10.3969/j.issn.1007-9572.2014.36.005.

[37] Lev EL, Daley KM, Conner NE, et al. An intervention to increase quality of life and self-care self-efficacy and decrease symptoms in breast cancer patients [J]. Sch Inq Nurs Pract, 2001, 15 (3): 277-294.

[38] Phillips SM, McAuley E. Social cognitive influences on physical activity participation in long-term breast cancer survivors [J]. Psychooncology, 2013, 22 (4): 783-791. DOI: 10.1002/pon.3074.

[39] Moon JA, Yoo CH, Kim MH, et al. Knowledge, Self-Efficacy, and Perceived Barriers on the Low-Iodine Diet among Thyroid Cancer Patients Preparing for Radioactive Iodine Therapy [J]. Clin Nutr Res, 2012, 1 (1): 13-22. DOI: 10.7762/cnr.2012.1.1.13.

[40] Ke YX, Liang ZQ, Huang FQ. Effect of self-efficacy training on quality of life of cancer patients undergoing chemotherapy [J]. Journal of Qiqihar Medical University, 2012, 33 (6): 791-792. DOI: 10.3969/j.issn.1002-1256.2012.06.063.

[41] Wang Li, Du YL, Jiang ZY, et al. Effect of self-efficacy intervention combined with medication on chemotherapy induced vomiting [J]. Journal of Nursing Science, 2015, 30 (13): 41-43. DOI: 10.3870/hlxzz.2015.13.041. 